\title{
ADJUNCTIONS AND COMONADS IN DIFFERENTIAL ALGEBRA
}

\author{
WILLIAM F. KEIGHER
}

\begin{abstract}
It is known that the construction of the ring of fractions $S^{-1} A$ of a commutative ring $A$ by a multiplicative subset $S$ of $A$ can be extended to the differential case. This means that for a given differential ring $(A, d)$, the differential ring of fractions of $(A, d)$ by $S$ is constructed simply by defining a derivation operator on $S^{-1} A$ in terms of the derivation operator $d$ on $A$. We seek to explain in the categorical setting of adjunctions and comonads the reasons for which this and other constructions can be extended to the differential case. A natural product of this investigation is the construction of the differential affine scheme of a differential ring.
\end{abstract}

1. Introduction. Stated simply, there are three points which explain why certain constructions involving commutative rings can be carried over to the differential case. These three points are adjunction, comonad and compatibility. The reader is referred to [9] for the necessary background on adjunctions and monads (to which comonads are dual). We add a few words to clarify each of these points.

By adjunction we mean that each of the constructions we consider is part of an adjunction, i.e., is an adjoint functor. This point will be made clearer as we discuss each example in $\S \S 3,4$ and 5 .

By comonad we mean that for each of the categories related to commutative rings there is a comonad on that category whose coalgebras are isomorphic to the differential analogue of that category. For example, the category Diff of differential rings is isomorphic to the category $\mathbf{C o m m}_{\Omega}$ of $\Omega$-coalgebras for a comonad $\Omega$ on the category Comm of commutative rings [7]. Since this example is of central importance for this paper, and since each of the other comonads we shall discuss is defined in terms of $\Omega$, we elaborate on this point below.

For the remainder of this paper we adopt the convention that all rings are commutative with unit and all ring homomorphisms preserve the unit. We also make frequent use of the notation $F$ : $\mathscr{A} \rightarrow \mathscr{B}: A \rightarrow F A: f \rightarrow F f$ when defining a functor $F: \mathscr{A} \rightarrow \mathscr{B}$ to describe its action upon objects $A \in \mathscr{A}$ and morphisms $f \in \mathscr{A}$.

The category Diff has as its objects differential rings which are pairs $(A, d)$ where $A$ is a ring and $d$ is a derivation operator on $A$, i.e., $d: A \rightarrow A$ is additive and satisfies the product rule $d(a b)=$ 
$d(a) b+a d(b)$ for any $a, b \in A$. A differential ring homomorphism $f:(A, d) \rightarrow\left(A^{\prime}, d^{\prime}\right)$ is a ring homomorphism $f: A \rightarrow A^{\prime}$ with $d^{\prime} f=f d$.

There is an adjunction $\langle U, G, \eta, \varepsilon\rangle:$ Diff $\rightarrow$ Comm where $U:$ Diff $\rightarrow$ Comm: $(A, d) \rightarrow A: f \rightarrow f$ is the forgetful functor. The right adjoint $G$ is defined by $G:$ Comm $\rightarrow$ Diff: $A \rightarrow\left(\omega A, \partial_{A}\right): f \rightarrow \omega f$, where for any ring $A, \omega A$ is defined as follows. The elements of $\omega A$ are countable sequences in $A$, i.e., of the form $\left(a_{n}\right)$ where $a_{n} \in A, n \in N=$ $\{0,1,2, \cdots\}$, with operations $\left(a_{n}\right)+\left(b_{n}\right)=\left(a_{n}+b_{n}\right)$ and $\left(a_{n}\right) \cdot\left(b_{n}\right)=\left(c_{n}\right)$, where $c_{n}=\sum_{k=0}^{n} C_{n, k} a_{k} b_{n-k}$. Here $C_{n, k}=n ! / k !(n-k) !$ denotes the usual binomial coefficient. The derivation operator $\partial_{A}$ on $\omega A$ is defined by $\partial_{A}\left(\left(a_{n}\right)\right)=\left(a_{n+1}\right)$, and for any ring homomorphism $f: A \rightarrow A^{\prime}$, $\omega f:\left(\omega A, \partial_{A}\right) \rightarrow\left(\omega A^{\prime}, \partial_{A^{\prime}}\right)$ is defined by $\omega f\left(\left(a_{n}\right)\right)=\left(f\left(a_{n}\right)\right)$. The unit $\eta:$ Diff $\rightarrow G U$ is, for any $(A, d) \in$ Diff and any $a \in A$, a differential ring homomorphism $\eta_{(A, d)}:(A, d) \rightarrow\left(\omega A, \partial_{A}\right)$ given by $\eta_{(A, d)}(\alpha)=\left(d^{(n)}(a)\right)$, where $d^{(n)}$ denotes the $n^{\text {th }}$ iterate of $d$ for $n \geqq 1$, and $d^{(0)}=i d_{A}$. The counit $\varepsilon: U G \rightarrow \mathbf{C o m m}$ is, for any $A \in \mathbf{C o m m}$ and any $\left(a_{n}\right) \in \omega A$, a ring homomorphism $\varepsilon_{A}: \omega A \rightarrow A$ given by $\varepsilon_{A}\left(\left(a_{n}\right)\right)=a_{0}$.

It follows from $[9, \mathrm{p} .135]$ that the adjunction $\langle U, G, \eta, \varepsilon\rangle$ : Diff $\rightarrow$ Comm defines a comonad $\Omega=(\omega, \varepsilon, \delta)=(U G, \varepsilon, U \eta G)$ on Comm. If $\mathrm{Comm}_{\Omega}$ denotes the category of $\Omega$-coalgebras and their morphisms, the cocomparison functor $\Phi:$ Diff $\rightarrow$ Comm $_{\Omega}$ which exists by [9, Theorem 1 , p. 138] is an isomorphism since $U$ satisfies the hypothesis of the dual of Beck's theorem [9, Theorem 1, p. 147]. We need not concern ourselves herein with the description of either the category $\operatorname{Comm}_{\Omega}$ or the isomorphism $\Phi$, but only with existence of the isomorphism $\Phi:$ Diff $\rightarrow \operatorname{Comm}_{\Omega}$.

Finally, by compatibility we mean that each of the adjunctions is compatible with the comonads involved in the sense that the right adjoint of each adjunction commutes with the comonads. As a consequence of the main result of $\S 2$, the adjunction extends to one between the coalgebras, which are seen to be the differential analogues of the categories in the original adjunction. It is in this sense that the constructions extend to the differential case.

2. Comonad adjunctions. Let $\mathscr{G}=(G, \varepsilon, \delta)$ and $\mathscr{G}^{\prime}=\left(G^{\prime}, \varepsilon^{\prime}, \delta^{\prime}\right)$ be comonads on $\mathscr{A}$ and $\mathscr{A}$ respectively. We say that $(S, \kappa)$ : $(\mathscr{A}, \mathscr{G}) \rightarrow\left(\mathscr{A}^{\prime}, \mathscr{G}^{\prime}\right)$ is a comonad functor if $S: \mathscr{A} \rightarrow \mathscr{A}^{\prime}$ is a functor and $\kappa: S G \rightarrow G^{\prime} S$ is a natural transformation such that $\varepsilon^{\prime} S \cdot \kappa=S \varepsilon$ and $\delta^{\prime} S \cdot \kappa=G^{\prime} \kappa \cdot \kappa G \cdot S \delta$.

If $(S, \kappa):(\mathscr{A}, \mathscr{G}) \rightarrow\left(\mathscr{A}^{\prime}, \mathscr{G}^{\prime}\right)$ and $\left(S^{\prime}, \kappa^{\prime}\right):\left(\mathscr{A}^{\prime}, \mathscr{G}^{\prime}\right) \rightarrow\left(\mathscr{A}^{\prime \prime}, \mathscr{G}^{\prime \prime}\right)$ are comonad functors, the composite $\left(S^{\prime}, \kappa^{\prime}\right) \cdot(S, \kappa)=\left(S^{\prime} S, \kappa^{\prime} S \cdot S^{\prime \prime} \kappa\right)$ : $(\mathscr{A}, \mathscr{G}) \rightarrow\left(\mathscr{A}^{\prime \prime}, \mathscr{G}^{\prime \prime}\right)$ is also a comonad functor. Hence there is a category Cmnd whose objects are pairs $(\mathscr{A}, \mathscr{G})$ where $\mathscr{A}$ is a category and $\mathscr{G}$ is a comonad on $\mathscr{A}$ and whose morphisms are the 
comonad functors defined above. If Cat denotes the category of all (small) categories, there is a functor Coalg: Cmnd $\rightarrow$ Cat: $(\mathscr{A}, \mathscr{G}) \rightarrow$ $\mathscr{A}_{S}:(S, \kappa) \rightarrow S_{\kappa}$, where for a comonad functor $(S, \kappa):(\mathscr{A}, \mathscr{C}) \rightarrow$ $\left(\mathscr{A}^{\prime}, \mathscr{G}^{\prime}\right), \quad S_{\kappa}: \mathscr{A}_{S} \rightarrow\left(\mathscr{A}^{\prime}\right)^{\prime}:(A, \alpha) \rightarrow\left(S A, \kappa_{A} \cdot S \alpha\right): f \rightarrow S f$. Other purely formal considerations in this direction may be found in [13].

We say that $\langle(S, \kappa),(T, \lambda), \sigma, \tau\rangle:(\mathscr{A}, \mathscr{G}) \rightarrow\left(\mathscr{A}^{\prime}, \mathscr{G}^{\prime}\right)$ is a comonad adjunction if $(S, \kappa):(\mathscr{A}, \mathscr{G}) \rightarrow\left(\mathscr{A}^{\prime}, \mathscr{G}^{\prime}\right)$ and $(T, \lambda):\left(\mathscr{A}^{\prime}, \mathscr{G}^{\prime}\right) \rightarrow$ $(\mathscr{A}, \mathscr{G})$ are comonad functors and $\sigma: \mathscr{A} \rightarrow T S$ and $\tau: S T \rightarrow \mathscr{A}^{\prime}$ are natural transformations such that

(i) $\langle S, T, \sigma, \tau\rangle: \mathscr{A} \rightarrow \mathscr{A}^{\prime}$ is an adjunction,

(ii) $\lambda S \cdot T \kappa \cdot \sigma G=G \sigma$, and

(iii) $G^{\prime} \tau \cdot \kappa T \cdot S \lambda=\tau G^{\prime}$.

We also say that an adjunction $\langle\bar{S}, \bar{T}, \bar{\sigma}, \bar{\tau}\rangle: \mathscr{\mathscr { A }} \rightarrow \overline{\mathscr{A}}^{\prime}$ extends another adjunction $\langle S, T, \sigma, \tau\rangle: \mathscr{A} \rightarrow \mathscr{A}^{\prime}$ by $\left(U, U^{\prime}\right)$ if $U: \mathscr{A} \rightarrow \mathscr{A}$ and $U^{\prime}: \mathscr{A}^{\prime} \rightarrow \mathscr{A}^{\prime}$ are functors such that $U^{\prime} \bar{S}=S U, U \bar{T}=T U^{\prime}$, $U \bar{\sigma}=\sigma U$ and $U^{\prime} \bar{\tau}=\tau U^{\prime}$, or equivalently if $\left(U, U^{\prime}\right)$ constitutes a map from the first adjunction to the second [9, Proposition 1, p. 97].

THEOREM 2.1. If $\langle(S, \kappa),(T, \lambda), \sigma, \tau\rangle:(\mathscr{A}, \mathscr{G}) \rightarrow\left(\mathscr{A}^{\prime}, \mathscr{G}^{\prime}\right)$ is a comonad adjunction, there are natural transformations $\bar{\sigma}, \bar{\tau}$ such that $\left\langle S_{k}, T_{\lambda}, \bar{\sigma}, \bar{\tau}\right\rangle: \mathscr{A}_{s} \rightarrow\left(\mathscr{A}^{\prime}\right)_{\xi^{\prime}}$ is an adjunction which extends $\langle S, T, \sigma, \tau\rangle: \mathscr{A} \rightarrow \mathscr{A}^{\prime}$ by $\left(U_{\mathscr{S}},\left(U^{\prime}\right)_{\mathscr{S}^{\prime}}\right)$.

Proof. This theorem follows from a theorem of Jean-Pierre Meyer [10, Theorem 2.2] in the case that $\mathscr{C}=\mathbf{C a t}_{*}$, the 2-category Cat with 2-cells reversed. In this case the natural transformation $\bar{\sigma}: \mathscr{A}_{\mathcal{G}} \rightarrow T_{\lambda} S_{k}$ may be defined for any $\mathscr{G}$-coalgebra $(A, \alpha)$ by $\bar{\sigma}_{(A, \alpha)}=$ $\sigma_{A}$, and similarly $\bar{\tau}$ may be defined for any $\mathscr{G}^{\prime}$-coalgebra $\left(A^{\prime}, \alpha^{\prime}\right)$ by $\bar{\tau}_{\left(A^{\prime}, \alpha^{\prime}\right)}=\tau_{A^{\prime}}$.

Let $\mathscr{G}=(G, \varepsilon, \delta)$ and $\mathscr{G}^{\prime}=\left(G^{\prime}, \varepsilon^{\prime}, \delta^{\prime}\right)$ be comonads on $\mathscr{A}$ and $\mathscr{A}^{\prime}$ respectively, and let $S: \mathscr{A} \rightarrow \mathscr{A}^{\prime}$ be a functor. We say that $S$ commutes with $\mathscr{G}$ and $\mathscr{G}^{\prime}$ if $G^{\prime} S=S G, \varepsilon^{\prime} S=S \varepsilon$ and $\delta^{\prime} S=S \delta$, or equivalently if the identity natural transformation id: $S G \rightarrow G^{\prime} S$ makes $\left(S\right.$, id): $(\mathscr{A}, \mathscr{G}) \rightarrow\left(\mathscr{A}^{\prime}, \mathscr{G}^{\prime}\right)$ a comonad functor.

THEOREM 2.2. Let $\mathscr{G}=(G, \varepsilon, \delta)$ and $\mathscr{G}^{\prime}=\left(G^{\prime}, \varepsilon^{\prime}, \delta^{\prime}\right)$ be comonads on $\mathscr{A}$ and $\mathscr{A}^{\prime}$ respectively, and let $\langle S, T, \sigma, \tau\rangle: \mathscr{A} \rightarrow \mathscr{A}^{\prime}$ be an adjunction. If $T$ commutes with $\mathscr{G}^{\prime}$ and $\mathscr{G}$, there is a natural transformation $\kappa: S G \rightarrow G^{\prime} S$ such that $\langle(S, \kappa),(T, \mathrm{id}), \sigma, \tau\rangle:(\mathscr{A}, \mathscr{G}) \rightarrow$ $\left(\mathscr{A}^{\prime}, \mathscr{G}^{\prime}\right)$ is a comonad adjunction. 
Proof. Define $\kappa$ to be the composite $\tau G^{\prime} S \cdot S \lambda^{-1} S \cdot S G \sigma$, where $\lambda=\mathrm{id}: T G^{\prime} \rightarrow G T$. One may then easily check that $\langle(S, \kappa),(T, \mathrm{id})$, $\sigma, \tau\rangle$ is a comonad adjunction.

REMARK. We observe that the conclusion of Theorem 2.2 remains valid if we replace the hypothesis that $T$ commutes with $\mathscr{G}^{\prime}$ and $\mathscr{G}$ by the hypothesis that $T$ commutes with $\mathscr{G}^{\prime}$ and $\mathscr{G}$ up to an isomorphism, i.e., there is a natural isomorphism $\lambda: T G^{\prime} \rightarrow G T$ which makes $(T, \lambda)$ a comonad functor. We do not need the added generality, however.

We now combine Theorems 2.1 and 2.2 to obtain the main result of this section. We will use this result in the subsequent sections to obtain the extensions of the constructions to the differential case.

Corollary 2.3. Let $\mathscr{G}$ and $\mathscr{G}^{\prime}$ be comonads on $\mathscr{A}$ and $\mathscr{A}^{\prime}$ respectively, and let $\langle S, T, \sigma, \tau\rangle: \mathscr{A} \rightarrow \mathscr{A}^{\prime}$ be an adjunction. If $T$ commutes with $\mathscr{G}^{\prime}$ and $\mathscr{G}$, there is an adjunction $\langle\bar{S}, \bar{T}, \bar{\sigma}, \bar{\tau}\rangle$ : $\mathscr{A}_{\mathscr{G}} \rightarrow\left(\mathscr{A}^{\prime}\right)_{\mathscr{S}^{\prime}}$ which extends $\langle S, T, \sigma, \tau\rangle: \mathscr{A} \rightarrow \mathscr{A}^{\prime}$ by $\left(U_{\mathscr{G}},\left(U^{\prime}\right)_{\mathscr{S}^{\prime}}\right)$.

REMARK. The dual of Corollary 2.3 was discovered independently by Peter Johnstone [6, Theorem 4].

3. Differential rings of fractions. The reader is referred to [2] for the basic results concerning rings of fractions. We begin by defining suitable categories for the adjunctions we develop in this section.

Let Comm' denote the category whose objects are pairs $(A, S)$ where $A$ is a ring and $S$ is a multiplicative subset of $A$. A morphism $f:(A, S) \rightarrow(B, T)$ in Comm' is a ring homomorphism $f: A \rightarrow B$ such that $f(S) \subset T$. Similarly let Diff' denote the category whose objects are pairs $((A, d), S)$ with $(A, d) \in$ Diff and $S$ a multiplicative subset of $A$, and whose morphisms are the obvious ones.

Proposition 3.1. There is an adjunction $\left\langle U^{\prime}, G^{\prime}, \eta^{\prime}, \varepsilon^{\prime}\right\rangle:$ Diff' $\rightarrow$ Comm', and the comonad $\Omega^{\prime}$ defined by this adjunction is such that $\left(\mathrm{Comm}^{\prime}\right)_{\Omega^{\prime}} \cong$ Diff' $^{\prime}$

Proof. The adjunction is defined in terms of the adjunction $\langle U, G, \eta, \varepsilon\rangle:$ Diff $\rightarrow$ Comm. The left adjoint $U^{\prime}$ is given by $U^{\prime}:$ Diff' $\rightarrow$ Comm': $((A, d), S) \rightarrow(A, S): f \rightarrow f$, while the right adjoint $G^{\prime}$ is defined by $\quad G^{\prime}: \mathbf{C o m m}^{\prime} \rightarrow$ Diff': $_{(A, S)} \rightarrow\left(\left(\omega A, \partial_{A}\right), S_{0}\right): f \rightarrow \omega f$, where $S_{0}=$ 
$\varepsilon_{A}^{-1}(S)=\left\{\left(a_{n}\right) \in \omega A: a_{0} \in S\right\}$. The unit $\eta^{\prime}:$ Diff $\rightarrow G^{\prime} U^{\prime}$ and counit $\varepsilon^{\prime}:$ $U^{\prime} G^{\prime} \rightarrow$ Comm' are given by $\left(\eta^{\prime}\right)_{((A, d), S)}=\eta_{(A, d)}$ and $\left(\varepsilon^{\prime}\right)_{(A, S)}=\varepsilon_{A}$. Observe that there are faithful functors $F: \operatorname{Comm}^{\prime} \rightarrow \operatorname{Comm}:(A, S) \rightarrow A: f \rightarrow f$ and $F^{\prime}:$ Diff' $\rightarrow$ Diff: $((A, d), S) \rightarrow(A, d): f \rightarrow f$ which forget the multiplicative subset and are such that $F^{\prime} G^{\prime}=G F, F U^{\prime}=U F^{\prime}, F^{\prime} \eta^{\prime}=$ $\eta F^{\prime}$, and $F \varepsilon^{\prime}=\varepsilon F$. It follows from this observation that $\left\langle U^{\prime}, G^{\prime}\right.$, $\left.\eta^{\prime}, \varepsilon^{\prime}\right\rangle:$ Diff $\rightarrow$ Comm' is an adjunction. The cocomparison functor

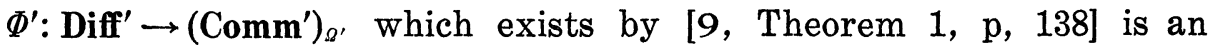
isomorphism since $U^{\prime}$ satisfies the hypothesis of the dual of Beck's theorem [9, Theorem 1, p. 147].

We now observe that the construction of $S^{-1} A$, the ring of

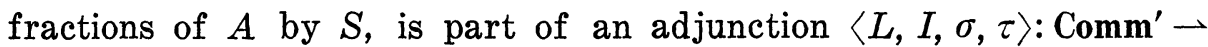
Comm. The left adjoint is defined by $L: \operatorname{Comm}^{\prime} \rightarrow \operatorname{Comm}:(A, S) \rightarrow$ $S^{-1} A: f \rightarrow f^{\prime}$, where for a morphism $f:(A, S) \rightarrow(B, T)$ in Comm', $f^{\prime}: S^{-1} A \rightarrow T^{-1} B$ is the unique ring homomorphism given by $f^{\prime}(a / s)=$ $f(a) / f(s)$ [2, Proposition 2, p. 77]. The right adjoint is given by $I$ : Comm $\rightarrow$ Comm' $^{\prime} A \rightarrow\left(A, A^{*}\right): f \rightarrow f$, where $A^{*}$ denotes the multiplicative set of invertible elements in $A$, i.e., the units of $A$.

LEMMA 3.2. An element $\left(a_{n}\right) \in \omega A$ is invertible if and only if $a_{0}$ is invertible in $A$, i.e., $(\omega A)^{*}=\varepsilon_{A}^{-1}\left(A^{*}\right)$.

Proof. Clearly if $\left(a_{n}\right) \in \omega A$ is invertible, then $\varepsilon_{A}\left(\left(a_{n}\right)\right)=a_{0}$ is invertible in $A$. Conversely suppose that $\left(a_{n}\right) \in \omega A$ is such that $a_{0}$ is invertible in $A$. Let $b_{0} \in A$ be such that $a_{0} b_{0}=1$, and for $n \geqq 1$ define $b_{n}$ inductively by

$$
b_{n}=-b_{0}\left(\sum_{k=1}^{n} C_{n, k} a_{k} b_{n-k}\right)
$$

One checks that $\left(a_{n}\right)\left(b_{n}\right)=1=\left(\delta_{0, n}\right)$, where $\delta_{0,0}=1$ and $\delta_{0, n}=0$ for $n \geqq 1$.

REMARK. Notice that Lemma 3.2 bears a strong resemblance to a theorem about formal power series rings, i.e., a power series $\sum_{n=0}^{\infty} a_{n} t^{n}$ is invertible in the ring $A[[t]]$ of formal power series in one variable with coefficients in $A$ if and only if the constant term $a_{0}$ is invertible in $A[8, \mathrm{p}$. 30]. The resemblance is no mere coincidence, however, since for any ring $A$ there is a natural differential ring homomorphism $\phi_{A}:(A[[t]], d / d t) \rightarrow\left(\omega A, \partial_{A}\right)$ defined by $\phi_{A}\left(\sum_{n=0}^{\infty} a_{n} t^{n}\right)=$ $\left(n ! a_{n}\right)$, where $d / d t$ denotes the usual termwise differentiation of power series. Moreover, if $A$ contains the ring of rationals, $\phi_{A}$ is an isomorphism. 
CoROLlary 3.3. There is an adjunction $\left\langle L^{\prime}, I^{\prime}, \sigma^{\prime}, \tau^{\prime}\right\rangle:$ Diff' $\rightarrow$ Diff which extends the adjunction $\langle L, I, \sigma, \tau\rangle: \mathbf{C o m m}^{\prime} \rightarrow$ Comm by $\left(U^{\prime}, U\right)$.

Proof. We first claim that $I$ commutes with $\Omega$ and $\Omega^{\prime}$. The equality $I \omega=\omega^{\prime} I$ follows from Lemma 3.2, and since $F$ : Comm' $\rightarrow$ Comm: $(A, S) \rightarrow A: f \rightarrow f$ is faithful, it suffices to show that $F I \varepsilon=$ $F \varepsilon^{\prime} I$ and $F I \delta=F \delta^{\prime} I$. But $F I=\mathrm{Comm}$, so that $F I \varepsilon=\varepsilon=\varepsilon F I=F \varepsilon^{\prime} I$, and similarly for the other equation. Now by Corollary 2.3 there is an adjunction $\langle\bar{L}, \bar{I}, \bar{\sigma}, \bar{\tau}\rangle:\left(\mathbf{C o m m}^{\prime}\right)_{\Omega^{\prime}} \rightarrow \mathbf{C o m m}_{\Omega}$ which extends $\langle L, I$, $\sigma, \tau\rangle:$ Comm' $^{\prime} \rightarrow$ Comm. The desired adjunction is induced by $\langle\bar{L}, \bar{I}$, $\bar{\sigma}, \bar{\tau}>$ and the isomorphisms Diff $\cong \mathrm{Comm}_{\Omega}$ and Diff $^{\prime} \cong\left(\mathrm{Comm}^{\prime}\right)_{\Omega^{\prime}}$.

REMARK. The functor $L^{\prime}$ : Diff' $\rightarrow$ Diff constructs the differential ring of fractions of $(A, d)$ by $S$. Since $U L^{\prime}=L U^{\prime}$, we see that $L^{\prime}((A, d), S)=\left(S^{-1} A, d^{\prime}\right)$ for some uniquely determined derivation operator $d^{\prime}$ on $S^{-1} A$. It is possible to show from what we have done that $d^{\prime}$ is the derivation operator defined for any $a \in A$ and $s \in S$ by

$$
d^{\prime}(a / s)=(s d(a)-a d(s)) / s^{2} .
$$

This is the usual quotient rule for the derivative of a fraction [1, p. 310], [3, p. 198], [8, p. 63].

4. Sheaves of differential rings. In this section we adopt the notation and conventions of [11]. In particular, if $X$ is a topological space and $\mathscr{A}$ is an $\mathscr{F}$-category, then $\mathscr{F}(H, \mathscr{A})$ denotes the category of sheaves in $\mathscr{A}$ over $X$.

If $S: \mathscr{A} \rightarrow \mathscr{B}$ is any continuous functor between $\mathscr{F}$-categories, there is an induced functor $S^{*}: \mathscr{F}(H, \mathscr{A}) \rightarrow \mathscr{F}(H, \mathscr{B}): F \rightarrow S F$ : $\alpha \rightarrow S \alpha$. This follows from the observation that if $S$ is continuous then $S$ preserves the equalizer property which characterizes the sheaves among the presheaves. In particular, if $S$ has a left adjoint, there is an induced $S^{*}$.

Proposition 4.1. For any topological space $X$ there is an adjunction $\left\langle U^{*}, G^{*}, \eta^{*}, \varepsilon^{*}\right\rangle: \mathscr{F}(X$, Diff $) \rightarrow \mathscr{F}(X, \mathrm{Comm})$, and the comonad $\Omega^{*}$ defined by this adjunction is such that $\mathscr{F}(X, \mathrm{Comm})_{\Omega^{*}} \cong$ $\mathscr{F}(X, \mathrm{Diff})$.

Proof. From the adjunction $\langle U, G, \eta, \varepsilon\rangle:$ Diff $\rightarrow$ Comm we see that $\mathrm{G}$ has a left adjoint $U$, and since $U$ is an algebraic functor it also has a left adjoint [12, Theorem 18.5.3. p. 238]. Hence by the 
observation made above there are induced functors $U^{*}$ and $G^{*}$. If we define $\eta^{*}$ and $\varepsilon^{*}$ by $\left(\eta^{*}\right)_{F}=\eta F$ and $\left(\varepsilon^{*}\right)_{F}=\varepsilon F$, then it is easy to see that $\left\langle U^{*}, G^{*}, \eta^{*}, \varepsilon^{*}\right\rangle$ is an adjunction. The cocomparison functor $\Phi^{*}: \mathscr{F}(X$, Diff $) \rightarrow \mathscr{F}(X, \text { Comm })_{\Omega^{*}}$ which exists by [9, Theorem 1, p. 138] is an isomorphism since $U^{*}$ satisfies the hypothesis of the dual of Beck's theorem [9, Theorem 1, p. 147].

Recall from [11, Theorem 5.1, p. 253] that if $\mathscr{A}$ is an $\mathscr{F}$-category and $f: X \rightarrow Y$ is a continuous map, there is an adjunction $\left\langle f^{*}, f_{*}\right.$, $\dot{\phi}, \dot{\psi}\rangle: \mathscr{F}(Y, \mathscr{A}) \rightarrow \mathscr{F}(X, \mathscr{A})$. The left adjoint $f^{*}$ is called the inverse image functor, while the right adjoint $f_{*}$ is called the direct image functor and is defined for any sheaf $F$ in $\mathscr{A}$ over $X$ and open set $V$ in $Y$ by $\left(f_{*} F\right)(V)=F\left(f^{-1}(V)\right)$.

Lemma 4.2. If $S: \mathscr{A} \rightarrow \mathscr{B}$ is a continuous functor between $\mathscr{F}$-categories and if $f: X \rightarrow Y$ is continuous, then $S^{*} f_{*}=f_{*} S^{*}$.

Proof. Let $F$ be a sheaf in $\mathscr{A}$ over $X$ and let $V$ be open in $Y$. Then $\left(S^{*} f_{*}\right)(F)(V)=S\left(\left(f_{*} F\right)(V)\right)=S F\left(f^{-1}(V)\right)=\left(f_{*} S F\right)(V)=$ $\left(f_{*} S^{*}\right)(F)(V)$.

Corollary 4.3. If $f: X \rightarrow Y$ is continuous, there is an adjunction $\left\langle f^{*}, f_{*}, \phi, \psi\right\rangle: \mathscr{F}(Y$, Diff $) \rightarrow \mathscr{F}(X$, Diff $)$ which extends the adjunction $\left\langle f^{*}, f_{*}, \dot{\phi}, \dot{\psi}\right\rangle: \mathscr{F}(Y, \mathrm{Comm}) \rightarrow \mathscr{F}(X, \mathrm{Comm})$ by $\left(U^{*}, U^{*}\right)$.

Proof. It follows from Lemma 4.2 that $f_{*}: \mathscr{F}(X$, Comm $) \rightarrow$ $\mathscr{F}(Y$, Comm $)$ commutes with the relevant $\Omega^{*}$ 's. Hence from Corollary 2.3 there is an adjunction $\left\langle\bar{f}^{*}, \bar{f}_{*}, \bar{\phi}, \bar{\psi}\right\rangle: \mathscr{F}(Y, \text { Comm })_{\Omega^{*}} \rightarrow$ $\mathscr{F}(X, \mathrm{Comm})_{\Omega^{*}}$ But $\mathscr{F}(\text { ?, Comm })_{\Omega^{*}} \cong \mathscr{F}($ ?, Diff $)$ by Proposition 4.1, which gives the desired adjunction.

REMARK. We observe from Corollary 4.3 that direct and inverse images of sheaves of differential rings over a topological space $X$ are constructed by forming direct or inverse images of the sheaves of the underlying rings, and the derivation operator on any section is then uniquely determined in terms of the derivation operator on the section of the original sheaf of differential rings.

We now observe that, for any complete and cocomplete category $\mathscr{A}$, topological space $X$ and $x \in X$, there is an adjunction $\left\langle S_{x}, K_{x}\right.$, $\sigma, \tau\rangle: \mathscr{F}(X, \mathscr{A}) \rightarrow \mathscr{A}$, where $S_{x}$ is the stalk functor, defined for any sheaf $F$ in $\mathscr{A}$ over $X$ by $S_{x} F=F_{x}=\lim F(U)$, the colimit taken over all open sets $U$ in $X$ which contain $\vec{x}$. The right adjoint $K_{x}$ is sometimes called the skyscraper sheaf functor, and is defined 
for any object $A$ and open set $U$ in $X$ by $K_{x} A(U)=A$ or 1 depending whether $x \in U$ or $x \notin U$, where 1 is the terminal object in $\mathscr{A}$.

COROLlary 4.4. For any topological space $X$ and any $x \in X$, there is an adjunction $\left\langle S_{x}, K_{x}, \sigma, \tau\right\rangle: \mathscr{F}(X$, Diff $) \rightarrow$ Diff which extends the adjunction $\left\langle S_{x}, K_{x}, \sigma, \tau\right\rangle: \mathscr{F}(X, \mathrm{Comm}) \rightarrow$ Comm by $\left(U^{*}, U\right)$.

Proof. The right adjoint $K_{x}:$ Comm $\rightarrow \mathscr{F}(X$, Comm $)$ can be seen to commute with $\Omega$ and $\Omega^{*}$, and hence by Corollary 2.3 there is an adjunction $\left\langle\bar{S}_{x}, \bar{K}_{x}, \bar{\sigma}, \bar{\tau}\right\rangle: \mathscr{F}(X, \mathbf{C o m m})_{\Omega^{*}} \rightarrow \mathbf{C o m m}_{\Omega}$. The desired adjunction follows from Proposition 4.1 and the isomorphism $\Phi$ : Diff $\rightarrow \mathbf{C o m m}_{2}$.

REMARK. It follows from Corollary 4.4 that the stalk of a sheaf of differential rings over a point $x \in X$ is a differential ring whose underlying ring is the stalk of the sheaf of the underlying rings over $x$, and the derivation operator on that ring is again uniquely determined.

5. Differential local ringed spaces and the differential affine scheme of a differential ring. In this section we show that an adjunction which is of fundamental importance in modern algebraic geometry is a comonad adjunction. The induced adjunction on the coalgebras gives the construction of the affine scheme of a differential ring. A second related adjunction yields the differential affine scheme of a differential ring.

For most of this section the notation and terminology will be consistent with that of [4]. We begin by stating several lemmas concerning local rings and local ring homomorphisms [2, p. 102]. $A^{*}$ will denote the units of the ring $A$.

Lemma 5.1. (i) Let $f: A \rightarrow B$ be a ring homomorphism such that $f^{-1}\left(B^{*}\right)=A^{*}$. Then if $B$ is local, so is $A$, and $f$ is a local ring homomorphism.

(ii) Let $A$ and $B$ be local rings and let $f: A \rightarrow B$ and $g: B \rightarrow A$ be ring homomorphisms with $g f=\mathrm{id}_{A}$. Then if $g$ is local, so is $f$.

Lemma 5.2. Let $\left(A_{\alpha}, \phi_{\beta \alpha}\right)$ be a directed system of rings, and let $A=\lim A_{\alpha}$ be the direct limit. Then the $A_{\alpha}^{*}$ form a directed system of sets with respect to restrictions of the $\phi_{\beta \alpha}$, and we have $A^{*}=$ $\lim _{\rightarrow} A_{\alpha}^{*}$

We will say that a sheaf $F$ in Comm over $X$ is local if for 
each $x \in X, F_{x}$ is a local ring, and a morphism $\alpha: F \rightarrow F^{\prime}$ of local sheaves in Comm over $X$ will be called local if $\alpha_{x}: F_{x} \rightarrow F_{x}^{\prime}$ is a local ring homomorphism for each $x \in X$. The following proposition says that the comonad $\Omega^{*}=\left(\omega^{*}, \varepsilon^{*}, \delta^{*}\right)$ on $\mathscr{F}(X, \mathrm{Comm})$ of Proposition 4.1 restricts to the subcategory of local sheaves and local morphisms.

Proposition 5.3. Let $F$ be a local sheaf in Comm over $X$. Then $\omega^{*} F$ is also a local sheaf in Comm over $X$, and $\varepsilon_{F}^{*}$ : $\omega^{*} F \rightarrow F$ and $\delta_{F}^{*}: \omega^{*} F \rightarrow \omega^{*} \omega^{*} F$ are local morphisms. Moreover, if $\alpha: F \rightarrow F^{\prime}$ is a local morphism, so is $\omega^{*} \alpha: \omega^{*} F \rightarrow \omega^{*} F^{\prime}$.

Proof. To show that $\omega^{*} F$ and $\varepsilon_{F}^{*}$ are both local, it suffices by Lemma 5.1 to show that $\left(\varepsilon_{F}^{*}\right)_{x}^{-1}\left(F_{x}^{*}\right)=\left(\omega^{*} F\right)_{x}^{*}$ for any $x \in X$. Taking all $\lim$ over the directed system $\mathscr{\mathscr { C }}_{x}$ of open sets $U$ in $X$ containing $x$, $\overrightarrow{\mathrm{we}}$ see that $\left(\varepsilon_{F}^{*}\right)_{x}^{-1}\left(F_{x}^{*}\right)=\left(\varepsilon_{F}^{*}\right)_{x}^{-1}(\lim F(U))^{*} \stackrel{(1)}{=}\left(\varepsilon_{F}^{*}\right)_{x}^{-1}\left(\lim F(U)^{*}\right) \stackrel{(2)}{=}$ $\lim \varepsilon_{F(U)}^{-1}\left(F(U)^{*}\right) \stackrel{(3)}{=} \lim \omega F(U)^{*} \stackrel{(1)}{=}(\lim \vec{\omega} F(U))^{*}=\left(\omega^{*} F\right)_{x}^{*}$. Here the

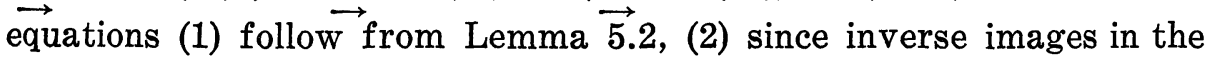
category of sets, Ens, are really pullbacks, hence finite limits, and that in Ens finite limits commute with colimits over directed sets ( $\mathscr{U}_{x}$ in this case) [9, Theorem 1, p. 211], and (3) from Lemma 3.2. Now from the comonad equations we have $\varepsilon_{\omega^{*} F}^{*} \cdot \delta_{F}^{*}=\mathrm{id}_{\omega^{*} F}$, and since $\varepsilon_{\omega^{*} F}^{*}$ is local by the above argument, Lemma 5.1 shows that $\delta_{F}^{*}$ is local. Finally, suppose that $\alpha: F \rightarrow F^{\prime}$ is a local morphism of local sheaves. Then since $\varepsilon_{F^{\prime}}^{*} \cdot \omega^{*} \alpha=\alpha \cdot \varepsilon_{F}^{*}$ and $\left(\omega^{*} F\right)_{x}^{*}=\left(\varepsilon_{F}^{*}\right)_{x}^{-1}\left(F_{x}^{*}\right)$, we see that $\left(\omega^{*} \alpha\right)_{x}^{-1}\left(\left(\omega^{*} F^{\prime}\right)_{x}^{*}\right)=\left(\omega^{*} F\right)_{x}^{*}$, so again by Lemma $5.1 \omega^{*} \alpha$ is local.

We will denote the category of local ringed spaces and their morphisms [4, p. 92-93] by Loc. We define a differential local ringed space to be a pair $\left(X, \mathscr{O}_{X}\right)$ where $X$ is a topological space and $\mathscr{O}_{X}$ is a sheaf in Diff on $X$ such that $U^{*} \mathscr{O}_{X}$ is local, i.e., $\left(U^{*} \mathscr{O}_{X}\right)_{x}=$ $U \mathscr{O}_{X, x}$ is a local ring for each $x \in X$. Observe that we are not yet requiring the maximal ideal in $U \mathscr{O}_{X, x}$ to be a differential ideal. If $\left(X, \mathscr{O}_{X}\right)$ and $\left(Y, \mathscr{O}_{Y}\right)$ are differential local ringed spaces, then $(\psi, \theta)$ : $\left(X, \mathscr{O}_{X}\right) \rightarrow\left(Y, \mathscr{O}_{Y}\right)$ is called a morphism of differential local ringed spaces if $\psi: X \rightarrow Y$ is continuous and $\theta: \mathscr{O}_{Y} \rightarrow \mathscr{O}_{X}$ is a local $\psi$ morphism of sheaves in Diff, i.e., $\theta: \mathscr{O}_{Y} \rightarrow \psi_{*} \mathcal{O}_{X}$ is a morphism in $\mathscr{F}\left(Y\right.$, Diff) such that $U^{*} \theta: U^{*} \mathscr{O}_{Y} \rightarrow U^{*} \psi_{*} \mathscr{O}_{X}=\psi_{*} U^{*} \mathscr{O}_{X}$ is a local morphism in $\mathscr{F}\left(Y\right.$, Comm). If $(\psi, \theta):\left(X, \mathscr{O}_{X}\right) \rightarrow\left(Y, \mathscr{O}_{Y}\right)$ and $\left(\psi^{\prime}, \theta^{\prime}\right)$ : $\left(Y, \mathscr{O}_{Y}\right) \rightarrow\left(Z, \mathscr{O}_{Z}\right)$ are morphisms of differential local ringed spaces, then their composite is given by $\left(\psi^{\prime}, \theta^{\prime}\right) \cdot(\psi, \theta)=\left(\psi^{\prime} \psi, \psi_{*}^{\prime} \theta \cdot \theta^{\prime}\right)$ : $\left(X, \mathscr{O}_{X}\right) \rightarrow\left(Z, \mathcal{O}_{Z}\right)$. The category of differential local ringed spaces 
will be denoted by Diff Loc.

We have seen that the adjunction $\langle U, G, \eta, \varepsilon\rangle:$ Diff $\rightarrow$ Comm defines the comonad $\Omega$ on Comm with $\operatorname{Comm}_{\Omega} \cong$ Diff, and similarly the adjunction $\left\langle U^{*}, G^{*}, \eta^{*}, \varepsilon^{*}\right\rangle: \mathscr{F}(X$, Diff $) \rightarrow \mathscr{F}(X$, Comm $)$ defines the comonad $\Omega^{*}$ on $\mathscr{F}(X, \mathrm{Comm})$ with $\mathscr{F}(X, \mathrm{Comm})_{\Omega^{*}} \cong \mathscr{F}(X, \mathrm{Diff})$. We extend the parallel to differential local ringed spaces.

THEOREM 5.4. There is an adjunction $\left\langle G^{0}, U^{0}, \varepsilon^{0}, \eta^{0}\right\rangle$ : Loc $\rightarrow$ Diff Loc, and the monad $\Omega^{\circ}$ defined by this adjunction is such that Loc $^{\Omega^{0}} \cong$ Diff Loc.

REMARK. We note that differential local ringed spaces are algebras for a monad, rather than coalgebras for a comonad as differential rings and sheaves of differential rings have been. This is due to the nature of the morphisms in Loc and Diff Loc, i.e., $(\psi, \theta):\left(X, \mathscr{O}_{X}\right) \rightarrow\left(Y, \mathscr{O}_{Y}\right)$ with $\theta: \mathscr{O}_{Y} \rightarrow \psi_{*} \mathscr{O}_{X}$ backwards (literally!).

Proof. The right adjoint is defined by $U^{0}:$ Diff Loc $\rightarrow$ Loc: $\left(X, \mathscr{O}_{X}\right) \rightarrow\left(X, U^{*} \mathcal{O}_{X}\right):(\psi, \theta) \rightarrow\left(\psi, U^{*} \theta\right)$, while the left adjoint is defined by $G^{0}:$ Loc $\rightarrow$ Diff Loc: $\left(X, \mathcal{O}_{X}\right) \rightarrow\left(X, G^{*} \mathcal{O}_{X}\right):(\psi, \theta) \rightarrow\left(\psi, G^{*} \theta\right)$. Note that by Proposition 5.3 if $\left(X, O_{X}\right)$ is a local ringed space then $U^{*} G^{*} \mathscr{O}_{X}=\omega^{*} \mathscr{O}_{X}$ is a local sheaf over $X$, and if $(\psi, \theta)$ is a morphism of local ringed spaces then $U^{*} G^{*} \theta=\omega^{*} \theta$ is a local morphism of sheaves over $Y$, so that $G^{0}$ is well defined. Define the unit $\varepsilon^{0}$ : Loc $\rightarrow U^{0} G^{0}$ and counit $\eta^{0}: G^{0} U^{0} \rightarrow$ Diff Loc by $\varepsilon_{\left(X, O_{X}\right)}^{0}=\left(\operatorname{id}_{X}, \varepsilon_{\sigma_{X}}^{*}\right)$ and $\eta_{\left(X, O_{X}\right)}^{0}=\left(\mathrm{id}_{X}, \eta_{\sigma_{X}}^{*}\right)$. Again by Proposition 5.3, $\varepsilon_{\sigma_{X}}^{*}$ is local and $\varepsilon_{U^{*} O_{X}}^{*} \cdot U^{*} \eta_{\sigma_{X}}^{*}=\mathrm{id}_{U^{*} O_{X}}$, so that by Lemma 5.1, $U^{*} \eta_{\sigma_{X}}^{*}$ is also local. It is clear that the adjunction equations for $\left\langle G^{0}, U^{0}, \varepsilon^{0}, \eta^{0}\right\rangle$ follow from those for $\left\langle U^{*}, G^{*}, \eta^{*}, \varepsilon^{*}\right\rangle$ and the (backward) composition of morphisms in both Loc and Diff Loc. It remains to show that the comparison functor $\Phi^{0}:$ Diff Loc $\rightarrow$ Loc $^{\Omega^{0}}$ which exists by [9, Theorem $1, \mathrm{p} .138]$ is an isomorphism, and for this we use Beck's theorem [9, Theorem 1, p. 147].

Let $\left(\psi_{i}, \theta_{i}\right):\left(X, \mathscr{O}_{X}\right) \rightarrow\left(Y, \mathscr{O}_{Y}\right), i=1,2$, be a parallel pair in Diff Loc for which $U^{0}\left(\psi_{i}, \theta_{i}\right)=\left(\psi_{i}, U^{*} \theta_{i}\right)$ has a split coequalizer in Loc, say

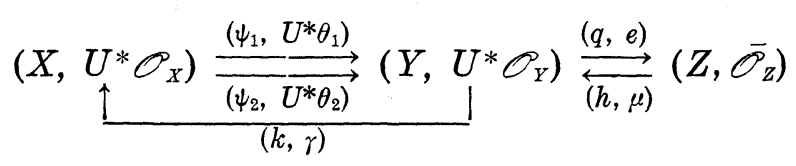

Using the rule $\left(f^{\prime}, \theta^{\prime}\right) \cdot(f, \theta)=\left(f^{\prime} f, f_{*}^{\prime} \theta \cdot \theta^{\prime}\right)$ for composition in Loc, it is not difficult to see that 


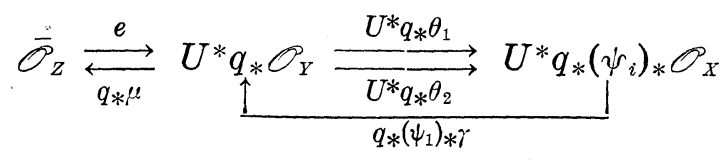

is a split equalizer in $\mathscr{F}(Z$, Comm). Now since the cocomparison functor $\Phi^{*}: \mathscr{F}(Z, \mathrm{Diff}) \rightarrow \mathscr{F}(Z, \mathrm{Comm})_{\Omega^{*}}$ is an isomorphism by Proposition 4.1, the dual of Beck's theorem implies that $U^{*}$ creates an equalizer for the parallel pair $q_{*} \theta_{1}, q_{*} \theta_{2}$ in $\mathscr{F}(Z$, Diff $)$. Hence $\bar{O}_{Z}=$ $U^{*} \mathcal{O}_{Z}$ and $e=U^{*} \theta$ for a unique $\mathscr{O}_{Z} \in \mathscr{F}\left(Z\right.$, Diff) and a unique $\theta: \mathscr{O}_{Z} \rightarrow$ $q_{*} \mathscr{O}_{Y}$ in $\mathscr{F}(Z$, Diff $)$, and $\theta$ is the equalizer of $q_{*} \theta_{1}$ and $q_{*} \theta_{2}$ in $\mathscr{F}(Z$, Diff $)$. It follows that $(q, \theta):\left(Y, \mathscr{O}_{Y}\right) \rightarrow\left(Z, \mathcal{O}_{Z}\right)$ is the coequalizer of $\left(\psi_{i}, \theta_{i}\right), i=1,2$, in Diff Loc, and from Beck's theorem we now conclude that $\Phi^{0}$ : Diff Loc $\rightarrow$ Loc $^{\Omega^{0}}$ is an isomorphism.

Recall now from [4] that there is an adjunction which enjoys a central role in modern algebraic geometry and which gives rise to the fundamental notion of the affine scheme of a ring. This adjunction will be denoted by $\langle$ Spec, $\Gamma, \theta, \rho\rangle:$ Comm $\rightarrow$ Loc $^{o p}$, where Loc $^{o p}$ is the category dual to Loc. Its left adjoint is the (contravariant) functor Spec: Comm $\rightarrow$ Loc $^{o p}$, which defines the affine scheme (Spec $(A)$, $\widetilde{A})$ of a ring $A[4,1.6 .1$, p. 209]. The right adjoint of the adjunction is the (contravariant) global sections functor $\Gamma:$ Loc $^{o p} \rightarrow$ Comm: $\left(X, \mathscr{O}_{X}\right) \rightarrow \mathscr{O}_{X}(X):(\psi, \theta) \rightarrow \Gamma(\theta)$. We also observe that the unit $\theta:$ Comm $\rightarrow \Gamma$ Spec of the adjunction is a natural isomorphism [4, 1.3.7, p. 199].

Corollary 5.5. There is an adjunction $\left\langle\operatorname{Spec}^{\prime}, \Gamma^{\prime}, \theta^{\prime}, \rho^{\prime}\right\rangle$ : Diff $\rightarrow$ Diff Loc ${ }^{o p}$ which extends the adjunction 〈Spec, $\left.\Gamma, \theta, \rho\right\rangle: \mathrm{Comm} \rightarrow$ Loc $^{o p}$, and $\theta^{\prime}:$ Diff $\rightarrow \Gamma^{\prime} \mathrm{Spec}^{\prime}$ is a natural isomorphism.

Proof. We first note that by the dual of Theorem 5.4 there is a comonad, which we shall denote by $\Omega^{0}$, on Loc $^{o p}$ such that $\left(\text { Loc }^{o p}\right)_{\Omega^{0}} \cong$ Diff Loc $^{o p}$. Furthermore, the right adjoint $\Gamma$ of the adjunction $\langle$ Spec, $\Gamma, \theta, \rho\rangle:$ Comm $\rightarrow$ Loc $^{\circ p}$ commutes with the comonads $\Omega^{0}$ and $\Omega$. By Corollary 2.3 there is an adjunction $\langle\overline{\text { Spec }}, \bar{\Gamma}, \bar{\theta}, \bar{\rho}\rangle: \mathbf{C o m m}_{\Omega} \rightarrow$ $\left(\mathbf{L o c}^{o^{p}}\right)_{\Omega^{0}}$ which extends $\langle$ Spec, $\Gamma, \theta, \rho\rangle: \mathbf{C o m m} \rightarrow$ Loc $^{o p}$, and the desired adjunction may be defined in terms of the adjunction $\langle\overline{\text { Spec }}, \bar{\Gamma}, \bar{\theta}, \bar{\rho}\rangle$ : $\operatorname{Comm}_{\Omega} \rightarrow\left(\text { Loc }^{o p}\right)_{\Omega^{0}}$ and the isomorphisms $\operatorname{Comm}_{\Omega} \cong$ Diff and $\left(\operatorname{Loc}^{o p}\right)_{\Omega^{0}} \cong$ Diff Loc ${ }^{o p}$. Finally, since $\left\langle\operatorname{Spec}^{\prime}, \Gamma^{\prime}, \theta^{\prime}, \rho^{\prime}\right\rangle$ extends $\langle\operatorname{Spec}, \Gamma, \theta, \rho\rangle$ by $\left(U, U^{0}\right)$ we see that $U \theta^{\prime}=\theta U$ is a natural isomorphism. But $U$ reflects isomorphisms, so that $\theta^{\prime}$ is a natural isomorphism.

For any differential $\operatorname{ring}(A, d), \operatorname{Spec}^{\prime}(A, d)=(\operatorname{Spec}(A),(\widetilde{A}, \widetilde{d}))$ is called the affine scheme of the differential ring $(A, d)$ and has many properties in common with the affine scheme of a ring. For 
example, we see from Corollary 5.5 that $\theta^{\prime}:$ Diff $\rightarrow \Gamma^{\prime}$ Spec' $^{\prime}$ is a natural isomorphism. This means that the differential coordinate ring of the affine scheme of any differential ring is naturally isomorphic to the differential ring, which in the non-differential case is a well known result. Moreover, one can easily show that the sheaf $(\widetilde{A}, \widetilde{d})$ on $\operatorname{Spec}(A)$ is such that for any $x \in \operatorname{Spec}(A),(\widetilde{A}, \widetilde{d})_{x} \cong$ $\left(A_{x}, d_{x}\right)$, where $A_{x}$ is the local ring of fractions $S^{-1} A$ with $S=A-j_{x}$ and $d_{x}$ is the derivation operator on $A_{x}$ defined by

$$
d_{x}(a / s)=(s d(a)-a d(s)) / s^{2}
$$

for any $a \in A, s \notin j_{x}$ (cf. $\S 3$ ).

Recall from [1, p. 315] that a local differential ring is a differential ring $(A, d)$ whose underlying ring $A$ is a local ring and whose maximal ideal $m_{A}$ is a differential ideal, i.e., $d\left(m_{A}\right) \subset m_{A}$ or equivalently $d^{-1}\left(A^{*}\right) \subset A^{*}$. We now define an LDR-space to be a differential local ringed space $\left(X, \mathcal{O}_{X}\right)$ such that for any $x \in X, \mathcal{O}_{X, x}$ is a local differential ring. The full subcategory of Diff Loc consisting of the LDR-spaces will be denoted by LDR.

Proposition 5.6. LDR is a coreflective subcategory of Diff Loc.

Proof. We show that the inclusion functor $K:$ LDR $\rightarrow$ Diff Loc has a right adjoint $D:$ Diff Loc $\rightarrow$ LDR. For any $\left(X, \mathcal{O}_{X}\right) \in$ Diff Loc, define $D\left(X, \mathscr{O}_{X}\right)=\left(X_{0}, \mathscr{O}_{X} \mid X_{0}\right)$, where $X_{0}=\left\{x \in X: \mathscr{O}_{X, x}\right.$ is a local differential ring $\}$ with the subspace topology and $\mathscr{O}_{X} \mid X_{0}$ is the restriction of $\mathscr{O}_{X}$ to $X_{0}$. Note that $\left(\mathscr{O}_{X} \mid X_{0}\right)_{x}=\mathscr{O}_{X, x}$ for any $x \in X_{0}$, so that $\left(X_{0}, \mathcal{O}_{X} \mid X_{0}\right) \in \mathbf{L D R}$. Now let $\left(i_{X}, \phi_{X}\right):\left(X_{0}, \mathcal{O}_{X} \mid X_{0}\right) \rightarrow\left(X, \mathcal{O}_{X}\right)$ denote the canonical injection, where $i_{X}: X_{0} \rightarrow X$ is the inclusion of the subspace and $\phi_{X}: \mathscr{O}_{X} \rightarrow\left(i_{X}\right)_{*}\left(\mathscr{O}_{X} \mid X_{0}\right)=\left(i_{X}\right)_{*}\left(i_{X}\right)^{*} \mathscr{O}_{X}$ is the unit of the adjunction $\left\langle\left(i_{X}\right)^{*},\left(i_{X}\right)_{*}, \phi, \psi\right\rangle: \mathscr{F}(X$, Diff $) \rightarrow \mathscr{F}\left(X_{0}\right.$, Diff $)$ from Corollary 4.3. To see that $D$ is a functor, let $(\psi, \theta):\left(X, \mathcal{O}_{X}\right) \rightarrow$ $\left(Y, O_{Y}\right)$ be a morphism of differential local ringed spaces. Then if $x \in X_{0}, \mathcal{O}_{X, x}$ is a local differential ring, so that $d_{x}^{-1}\left(\mathcal{O}_{X, x}^{*}\right) \subset \mathcal{O}_{X, x}^{*}$, where $d_{x}$ denotes the derivation operator of $\mathcal{O}_{X, x}$. Since $\theta_{x}^{\sharp}: \mathcal{O}_{Y, \psi(x)} \rightarrow \mathcal{O}_{X, x}$ is a differential ring homomorphism it follows that $\left(\theta_{x}^{\sharp}\right)^{-1} d_{x}^{-1}\left(\mathcal{O}_{X, x}^{*}\right)=$ $d_{\bar{\psi}_{(x)}^{1}}^{-1}\left(\theta_{x}^{\sharp}\right)^{-1}\left(\mathcal{O}_{X, x}^{*}\right) \subset\left(\theta_{x}^{\#}\right)^{-1}\left(\mathcal{O}_{X, x}^{*}\right)$, and since $(\psi, \theta)$ is a morphism in Diff Loc we see that $\left(\theta_{x}^{\sharp}\right)^{-1}\left(\mathcal{O}_{X, x}^{*}\right)=\mathscr{O}_{Y, \psi(x)}^{*}$. Hence $d_{\psi(x)}^{1}\left(\mathcal{O}_{Y, \psi(x)}^{*}\right) \subset \mathcal{O}_{Y, \psi(x)}^{*}$, so that $\mathcal{O}_{Y, \psi(x)}$ is a local differential ring and $\psi(x) \in Y_{0}$. Therefore there exists a unique continuous $\psi_{0}: X_{0} \rightarrow Y_{0}$ such that $\psi \cdot i_{X}=i_{Y} \cdot \psi_{0}$. One checks that $\theta: \mathscr{O}_{Y} \rightarrow \psi_{*} \mathcal{O}_{X}$ also restricts properly to give $\theta \mid Y_{0}$ : $\mathscr{O}_{Y} \mid Y_{0} \rightarrow\left(\psi_{0}\right)_{*}\left(\mathcal{O}_{X} \mid X_{0}\right)$ by observing that $\mathscr{O}_{X}\left|X_{0}=\left(i_{X}\right)^{*} \mathcal{O}_{X}, \mathcal{O}_{Y}\right| Y_{0}=$ $\left(i_{Y}\right) * \mathscr{O}_{Y}$ and $\psi \cdot i_{X}=i_{Y} \cdot \psi_{0}$. Hence $D$ is a functor, and clearly $D K=$ $\mathrm{id}_{\text {LDR. }}$. There is also a natural transformation $i: K D \rightarrow \mathrm{id}_{\text {Diff Loc }}$ with 
components $i_{\left(X, O_{X}\right)}=\left(i_{X}, \phi_{X}\right):\left(X_{0}, \mathscr{O}_{X} \mid X_{0}\right) \rightarrow\left(X, \mathscr{O}_{X}\right)$ as above. Finally, one checks that $\langle K, D$, id, $i\rangle: \mathbf{L D R} \rightarrow$ Diff Loc is the desired adjunction.

COROLLARY 5.7. There is an adjunction $\left\langle\operatorname{Spec}_{D}, \Gamma_{D}, \theta_{D}, \rho_{D}\right\rangle$ : Diff $\rightarrow \mathbf{L D R}^{o p}$.

Proof. By the dual of Proposition 5.6 there is an adjunction $\langle D, K, i, \mathrm{id}\rangle:$ Diff $\mathbf{L o c}^{o p} \rightarrow \mathbf{L D R}^{o p}$, and by Corollary 5.5 there is an adjunction $\left\langle\right.$ Spec' $\left.^{\prime}, \Gamma^{\prime}, \theta^{\prime}, \rho^{\prime}\right\rangle:$ Diff $\rightarrow$ Diff Loc ${ }^{o p}$. The two adjunctions can be composed [9, Theorem 1, p. 101] to give the adjunction $\left\langle\operatorname{Spec}_{D}, \Gamma_{D}, \theta_{D}, \rho_{D}\right\rangle=\left\langle D \mathrm{Spec}^{\prime}, \Gamma^{\prime} K, \Gamma^{\prime} i \mathrm{Spec}^{\prime} \cdot \theta^{\prime}\right.$, id $\left.\cdot D \rho^{\prime} K\right\rangle:$ Diff $\rightarrow$ $\mathbf{L D R}^{o p}$.

REMARK. We observe that the adjunction $\left\langle\operatorname{Spec}_{D}, \Gamma_{D}, \theta_{D}, \rho_{D}\right\rangle$ : Diff $\rightarrow \mathbf{L D R}^{o p}$ does not extend $\langle$ Spec, $\Gamma, \theta, \rho\rangle: \mathbf{C o m m} \rightarrow \mathbf{L o c}^{o p}$, and more importantly that $\theta_{D}$ : Diff $\rightarrow \Gamma_{D}$ Spec $_{D}$ is not a natural isomorphism. The latter observation follows since $\theta_{D}=\Gamma^{\prime} i \mathrm{Spec}^{\prime} \cdot \theta^{\prime}$, and while $\theta^{\prime}$ : Diff $\rightarrow \Gamma^{\prime}$ Spec $^{\prime}$ is a natural isomorphism, $i$ is not an isomorphism.

The adjunction of Corollary 5.7 has considerable significance for differential algebraists, since the basic objects that one usually considers in differential algebraic geometry do not involve all the prime ideals in a differential ring but rather only the prime differential ideals. We claim that for any differential ring $(A, d)$, $\operatorname{Spec}_{D}(A, d)$ is exactly a basic object. By definition, $\operatorname{Spec}_{D}(A, d)=$ $D \operatorname{Spec}^{\prime}(A, d)=D(\operatorname{Spec}(A),(\widetilde{A}, \widetilde{d}))=\left(\operatorname{Spec}(A)_{0},(\widetilde{A}, \widetilde{d}) \mid \operatorname{Spec}(A)_{0}\right)$, where $\operatorname{Spec}(A)_{0}=\left\{x \in \operatorname{Spec}(A):(\widetilde{A}, \widetilde{d})_{x} \text { is a local differential ring }\right\}_{\text {. But }}$ $(\widetilde{A}, \widetilde{d})_{x}=\left(A_{x}, d_{x}\right)$ is a local differential ring if and only if $m_{x}=j_{x} A_{j_{x}}$, the maximal ideal of $A_{x}$, is a differential ideal, and this is so if and only if $j_{x}$ is a differential ideal. Hence Spec $(A)_{0}$ consists of the prime differential ideals of $(A, d)$, and we denote this subspace of $\operatorname{Spec}(A)$ by $\operatorname{Spec}_{D}(A)$.

We will call $\operatorname{Spec}_{D}(A, d)=\left(\operatorname{Spec}_{D}(A),(\widetilde{A}, \widetilde{d})^{*}\right)$ the differential affine scheme of the differential ring $(A, d)$. We observe that since $\operatorname{Spec}_{D}$ is part of an adjunction, $\left(\operatorname{Spec}_{D}(A),(\widetilde{A}, \widetilde{d})^{*}\right)$ has many properties in common with the affine scheme $(\operatorname{Spec}(A),(\widetilde{A}, \widetilde{d}))$ defined earlier. Moreover, these differential affine schemes will be the basic objects used to define differential schemes which are the differential analogue of schemes. The definitions and important properties of differential schemes will be the topic of a separate paper. 


\section{REFERENCES}

1. P. Blum, Complete models of differential fields, Trans. Amer. Math. Soc., 137 (1969), 309-325.

2. N. Bourbaki, Algébre Commutative, Éléments de Mathematique, Vol. 27, Hermann, Paris, 1961.

3. H. Gorman, Radical regularity in differential rings, Canad. J. Math., 23 (1971), 197-201.

4. A. Grothendieck and J. Dieudonné, Éléments de Géométrie Algébrique I, SpringerVerlag, Berlin, 1971.

5. I. Kaplansky, An introduction to differential algebra, Actualités Sci. Indust., 1251 (1957), 9-63.

6. P. Johnstone, Adjoint lifting theorems for categories of algebras, to appear in Bull. London Math. Soc.

7. W. Keigher, Symmetric monoidal comonads and differential algebra, to appear.

8. E. Kolchin, Differential Algebra and Algebraic Groups, Academic Press, New York, 1973.

9. S. Mac Lane, Categories for the Working Mathematician, Graduate Texts in Mathematics 5, Springer-Verlag, New York, 1971.

10. J.-P. Meyer, Induced functors on categories of algebras, to appear in Math. Z.

11. B. Mitchell, Theory of Categories, Academic Press, New York, 1965.

12. H. Schubert, Kategorien II, Springer-Verlag, Berlin, 1970.

13. R. Street, Formal theory of monads, J. Pure Appl. Algebra, 2 (1971), 149-168.

Received August 22, 1974 and in revised form February 20, 1975. A portion of the research of the present paper was completed as a part of the author's doctoral dissertation done at the University of Illinois at Urbana-Champaign under the direction of Professor John W. Gray. The remainder of the research and the writing was completed while the author was at Southern Illinois University at Carbondale.

The University of Tennessee 